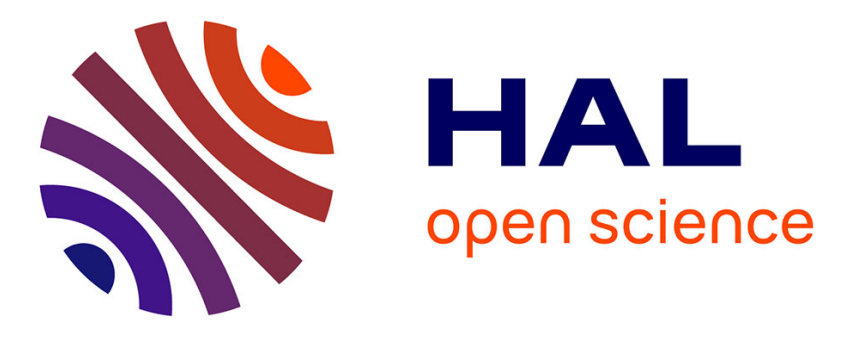

\title{
High peak and average power delivery from Fully-Aperiodic Large-Pitch-Fiber lasers (Invited)
}

Philippe Roy, Raphaël Jamier, Marie-Alicia Malleville, Baptiste Leconte, Romain Dauliat, Anka Schwuchow, Katrin Wondraczek, Julien Didierjean

\section{- To cite this version:}

Philippe Roy, Raphaël Jamier, Marie-Alicia Malleville, Baptiste Leconte, Romain Dauliat, et al.. High peak and average power delivery from Fully-Aperiodic Large-Pitch-Fiber lasers (Invited). 2019 IEEE 8th International Conference on Advanced Optoelectronics and Lasers (CAOL), Sep 2019, Sozopol, Bulgaria. pp.5-9, 10.1109/CAOL46282.2019.9019495 . hal-02503342

\section{HAL Id: hal-02503342 \\ https://hal.science/hal-02503342}

Submitted on 10 Dec 2020

HAL is a multi-disciplinary open access archive for the deposit and dissemination of scientific research documents, whether they are published or not. The documents may come from teaching and research institutions in France or abroad, or from public or private research centers.
L'archive ouverte pluridisciplinaire HAL, est destinée au dépôt et à la diffusion de documents scientifiques de niveau recherche, publiés ou non, émanant des établissements d'enseignement et de recherche français ou étrangers, des laboratoires publics ou privés. 


\section{High peak and average power delivery from Fully- Aperiodic Large-Pitch-Fiber lasers}

\author{
Philippe ROY \\ University of Limoges, CNRS \\ XLIM, UMR 7252 \\ 87000 Limoges, FRANCE \\ philippe.roy@xlim.fr \\ Marie-Alicia MALLEVILLE \\ Eolite Systems \\ 11 Avenue de Canteranne \\ 33600 Pessac, France \\ marie-alicia.malleville@xlim.fr \\ Anka SCHWUCHOW \\ Leibniz Institute of Photonic \\ Technology \\ Albert-Einstein-Straße 9 \\ 07745 Jena, Germany \\ anka.schwuchow@1eibniz-ipht.de
}

\author{
Raphael JAMIER \\ University of Limoges, CNRS \\ XLIM, UMR 7252 \\ 87000 Limoges, FRANCE \\ raphael.jamier@xlim.fr \\ Baptiste LECONTE \\ University of Limoges, CNRS \\ XLIM, UMR 7252 \\ 87000 Limoges, FRANCE \\ baptiste.leconte@xlim.fr \\ Katrin WONDRACZEK \\ Leibniz Institute of Photonic \\ Technology \\ Albert-Einstein-Straße 9 \\ 07745 Jena, Germany \\ katrin.wondraczek@leibniz-ipht.de
}

\author{
Marie-Alicia MALLEVILLE \\ University of Limoges, CNRS \\ XLIM, UMR 7252 \\ 87000 Limoges, FRANCE \\ marie-alicia.malleville@xlim.fr
}

Romain DAULIAT

University of Limoges, CNRS

XLIM, UMR 7252

87000 Limoges, FRANCE

romain.dauliat@xlim.fr

\author{
Julien DIDIERJEAN \\ Eolite Systems \\ 11 Avenue de Canteranne \\ 33600 Pessac, France \\ didierjeanj@esi.com
}

\begin{abstract}
In this invited communication, we perform a review on Fully-Aperiodic Large Pitch Fibers. Major advantages and drawbacks of this design are presented first as well as the synthesis method, which enables the success of their fabrication. Some laser experiments are then detailed in order to prove the relevance of the aperiodic fiber concept. Very last experimental results and some prospects will be finally shown during the conference.
\end{abstract}

Keywords-Fiber-laser, Advanced-fibers, Singlemode VLMA, Microstructured fibers, High-peak power.

\section{INTRODUCTION}

By proposing new paradigms in the field of light confinement and amplification, Photonic Crystal Fibres (PCF) proposed by Knight et al. more than 20 years ago [1], have paved the way toward power scaling in fibre lasers and amplifiers. For pulsed fiber lasers especially, the achievement of larger and larger mode areas while maintaining a transverse single-mode operation have driven a constant progression of the state-of-the-art. Pulse energy, average power and peak power have simultaneously grown. Among others, Leakage Chanel Fibres (LCF) [2], rod type PCF [3], Distributed Mode Filtering (DMF) fibres [4] and Large-Pitch Fibres (LPFs) [5] have particularly stood the attention of international scientific community in the field of Very Large Mode Area (VLMA) optical fibres for lasers. For instance, up to $22 \mathrm{GW}$ (200 fs) of peak power (respectively $314 \mathrm{~kW} / 25 \mathrm{ps}$ ) have been demonstrated in LPFs [6] (resp. in DMF fibres [7]). However, since 2011, the performances delivered by industrial pulsed VLMA fibre lasers available on the market appeared to be mostly limited by a threshold-like phenomenon, the transverse mode instabilities (TMI). This phenomenon which results in a significant degradation of the beam quality due to temporal modal beating has been extensively studied, both theoretically [8,9] and experimentally [10-13]. Indeed, when propagation properties (due to the structure of the guide itself or due to a heat-load induced guide) enable the coexistence of several transverse modes, these different transverse modes induce periodical modifications of the refractive index along the fibre that create unexpected power coupling between modes, ultimately leading to the modal instabilities [8]. To overcome these limitations, we have proposed, since 2013, a new design of PCF, so called Fully-Aperiodic Large-Pitch Fibres (FALPF) [14]. Section 2 of this manuscript is dedicated to the general presentation of the FA-LPF concept and its advantages. We will also quickly describe the fabrication method including the synthesis of the different doped-silica materials. The impact of fabrication defects will be clearly highlighted in this section thanks to basic laser characterizations, evaluation and comparison of TMI thresholds of different fibres. Section 3 will be devoted to the presentation of quasi-industrial grade laser sources and burnin evaluation. Finally, in the last section, some prospects are introduced. A first demonstration of thermal precompensation of the index profile is described whereas other features (such as bent resistive, PZ or short length designs) will be discussed during the conference.

\section{THE FA-LPF CONCEPT}

\section{A. Theoretical presentation of the FA-LPF design}

As mentioned earlier, the main limitations of large-core fibre lasers and amplifiers are related to TMI $[8,15]$. This phenomenon is all the more important as several modes can interact with the gain area. Thus, thermal loading and photodarkening, which can accentuate the confinement of the High Order Modes (HOM), consequently decrease the TMI threshold [15]. When we started the work on the FALPF, our aim was to reinforce the singlemodeness of the proposed fibres, whether the fibre is cold or suffer a significant thermal loading. Another major consideration was to propose a realistic solution compatible with existing manufacturing processes. We also chose to limit the degrees of freedom in the design by taking into account practical considerations such as reducing manufacturing costs, achieving all-solid fiber to simplify splicing and fiber implementation in all-fiber laser sources. 1D and 2D index mapping are displayed in figure 1. 


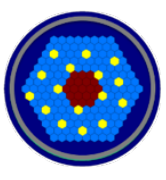

(a)

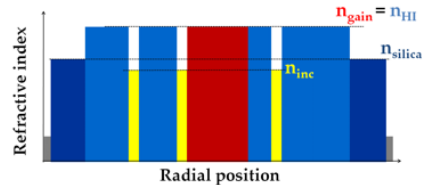

(b)

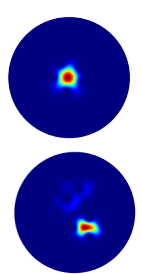

(c)
Fig. 1. Schematic representation of (a) the cross-section and (b) refractive index profile of the proposed FA-LPF. Red and blue areas represent respectively the $\mathrm{Yb}$-doped core $\left(\mathrm{n}_{\text {gain }}\right)$ and the passive indexmatched high-index background cladding $\left(\mathrm{n}_{\mathrm{HI}}=\mathrm{n}_{\text {gain }}\right)$. Low-index inclusions stands in yellow. (c) Near field intensity distribution for the fundamental mode (top) and the $\mathrm{LP}_{11}$-like mode (bottom) in the FA-LPF.

In FA-LPF, the confinement principle of light is based on the modal sieve concept like it is for LCFs [2] and LPFs [5]. Unlike the latter or more generally the LMA fibers, FA-LPF design has grown around a uniformly rare-earth-doped core (red area in Fig.1) [17]. This frees a lot of constraints during glass synthesis as rare-earth ions and additional passive dopant elements can be used to optimize the material optical efficiency regardless of the control over the refractive index. Compared to filamented core, the whole core area is uniformly doped and for a given pump absorption level, the local rareearth concentration is lower, which decreases the impact of photodarkening $[17,18]$. Finally, it also significantly simplifies the fabrication process and potentially decreases the production cost of the material considering the suppression of the multiple-step, stack and draw process. However, a second high-index material (this one being passive) needs to be synthesized to be used as background material in the cladding. We will show later the influence of any refractive index mismatch on the guidance properties of the fiber; the refractive indices of the two materials are supposed to be strictly identical in the theoretical concept. The modal content of the central core is therefore controlled by a set of solid low index inclusions displayed in an aperiodic way in the passive high-index background cladding material (see Fig.1(a)). These materials are represented respectively in yellow and blue in Fig.1(a). In order to make our design compatible with standard fabrication methods (stack and draw method), the external part of the structure is made of pure silica and includes an air-cladding to confine high-numerical-aperture high-power pump waves.

In such a structure, the fundamental mode is very well confined in the active core and is not affected by the disorder of the low-index inclusions in the cladding (Fig.1(c-up)). On the contrary, the aperiodicity of the low index pattern in the cladding improves the HOMs delocalization out of the gain area. For example, the second mode ( $\mathrm{LP}_{11}$-like) is strongly distorted and highly delocalized as evidenced by his intensity distribution shows in figure 1(c-bottom). As these active fibers have double cladding structures to ensure the propagation of high numerical aperture pump power, confinement losses are not discriminating. The structure evaluation relies on the overlap factor of each mode with the active area, the modal selection being operated by the gain filtering under amplifier/laser operation. To illustrate this purpose, in figure 2, the overlap factors of the 300 first modes are plotted and compared for a LPF (Fig. 2.a) and a FA-LPF (Fig.2.b). In both cases, mode areas can be varied without significant impact. Depending on the structure, the most competitive High Order Mode is not systematically the $\mathrm{LP}_{11}$ or LP $\mathrm{P}_{11}$-like mode.

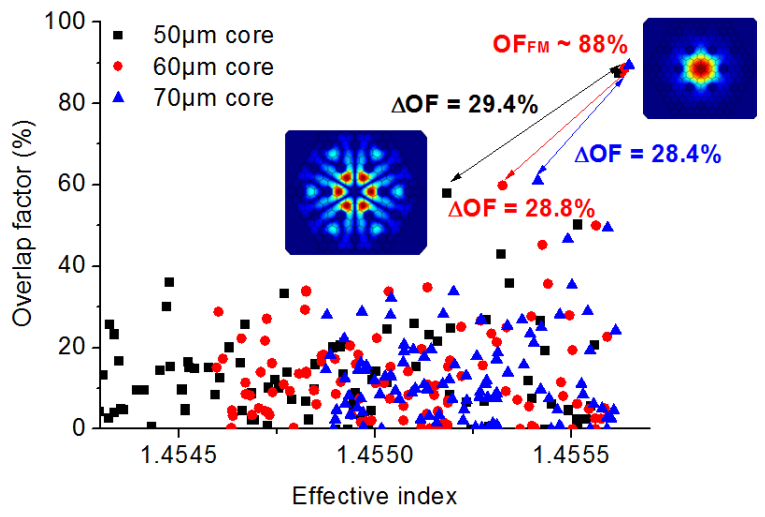

(a)

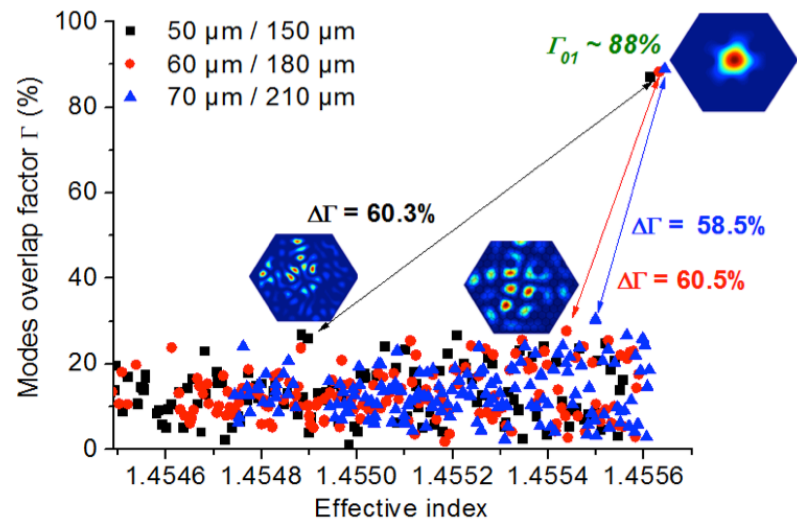

(b)

Fig.2. Overlap factors calculated for the 300 firsts modes in (a) a standard LPF and (b) a FA-LPF. The core size can be varied from $50 \mu \mathrm{m}$ to $70 \mu \mathrm{m}$ without significant change.

For fundamental mode, figures 2 (a) and 2(b) show that an overlap factor higher than $80 \%$ is obtained for both structures and this reflects a potential good amplification efficiency. On the contrary, for higher order modes, by breaking their resonance in the cladding, the overlap factors are significantly lower, minimizing their impact in the TMI process.

\section{B. Fabrication method of the FA-LPF}

As mentioned before, such a design does not make sense without a relevant manufacturing process. For such large core areas and as far as stack and draw process is forebode for the fabrication, it has been necessary to identify and develop a method suitable to produce volumes of homogeneously and very accurately controlled doped silica. The sintering and vitrification of doped silica powders known as REPUSIL [19] can fulfill these requirements as volume 40 to 100 times larger than that produced by MCVD can be synthetized. On the other hand, the levels of control on the refractive index (standard deviation to the target value and homogeneity) that can be obtained with CVD processes are in the range of $10^{-4}$ (gas phase doping technique) to $10^{-3}$ (standard solution doping 
technique). As mentioned earlier, the control of the refractive index values of synthetized materials is essential for all the LMA fiber structures. The figure 3 shows that, for $80-100 \mu \mathrm{m}$ core diameters, the required level of control on the refractive index contrast between core and inner cladding material cannot goes beyond few $10^{-5}$, which is also below the detection limit of commercial refractive index measurement devices $\left(10^{-4}\right.$ for IFA [20]). During the development of the fabrication process, we have fabricated several couples of materials (one active and one passive) slowly improving the index matching between each other and consequently slowly increasing our ability to fabricate larger and larger cores (see figure 3 ).

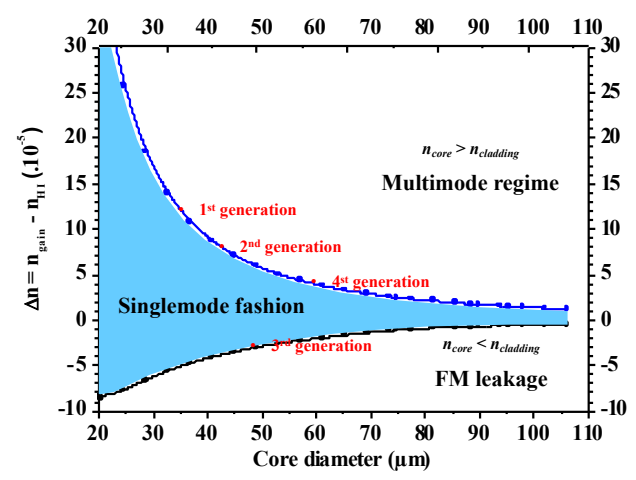

Fig.3. Evolution of the refractive index difference tolerated to ensure an effective transverse single-mode behavior at an operating wavelength of $1.03 \mu \mathrm{m}$ in regard to the core diameter of the fiber.

\section{Impact of the refractive index contrast of materials on the TMI threshold}

For each couple of materials, several test fibers have been drawn with different core diameters so that we were able to conduct a study on TMI threshold based on a significant batch of fiber samples. As mentioned earlier, beyond the fiber itself, a lot of parameters related to the laser architecture, in the broad sense of the term, can influence the TMI threshold. It is for example well known that a narrow linewidth Fabry-Perot laser source operated in continuous wave regime is particularly drastic. In order to observe the TMI threshold with moderate pump power levels, we have used such a laser architecture and its schematic representation is shown in figure 4 .

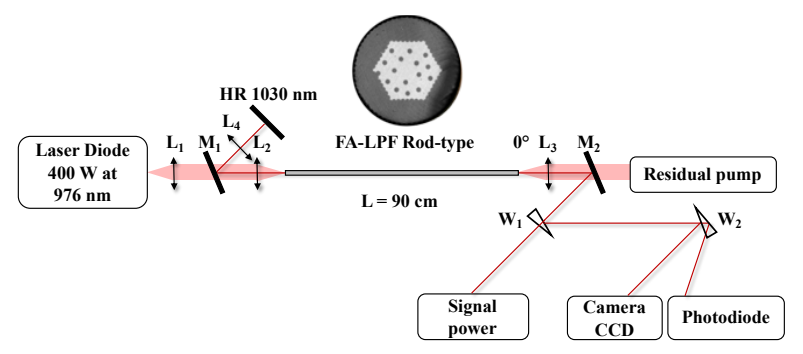

Fig.3. Schematic representation of the experimental laser setup used for TMI threshold investigations and fiber classification. HR1030 is a high-reflectivity mirror at $1030 \mathrm{~nm}$; $\mathrm{M} 1$ and $\mathrm{M} 2$ are $22.5^{\circ}$ dichroic mirrors (HR1030/HT976); L1, L2, L3, and L4 are lenses; W1 and W2 are wedge plates. Insert: microscope image of the $90 \mathrm{~cm}$ long FA-LPF under test.

For a given refractive index contrast of $8.10^{-5}$, Table 1 gives the evolution of the experimentally measured TMI threshold versus the core diameter. For a given core diameter close to $60 \mu \mathrm{m}$, Table 2 gives the evolution of the experimentally measured TMI threshold versus the refractive index contrast.

TABLE I. EXPERIMENTALLY MEASURED TMI THRESHOLD VERSUS CORE DIAMETER FOR A FA-LPF EXHIBITING A $8.10^{-5}$ REFRACTIVE INDEX CONTRAST

\begin{tabular}{|c|c|}
\hline Core diameter $(\boldsymbol{\mu m})$ & Measured TMI Threshold $(\mathbf{W})$ \\
\hline 58 & $100-105$ \\
\hline 84 & $80-85$ \\
\hline 100 & $55-60$ \\
\hline
\end{tabular}

TABLE II. EXPERIMENTALLY MEASURED TMI THRESHOLD VERSUS REFRACTIVE INDEX CONTRAST FOR A 60 $\mu \mathrm{M}$ MODE FIELD DIAMETER FA-LPF

\begin{tabular}{|c|c|c|}
\hline $\begin{array}{c}\text { Refractive } \\
\text { index } \\
\text { contrast } \Delta \mathbf{n}\end{array}$ & $\begin{array}{c}\text { Core diameter } \\
(\boldsymbol{\mu m})\end{array}$ & $\begin{array}{c}\text { Measured TMI Threshold } \\
(\mathbf{W})\end{array}$ \\
\hline $13.10^{-5}$ & 65 & $55-60$ \\
\hline $8.10^{-5}$ & 58 & $100-105$ \\
\hline $4.10^{-5}$ & 62 & $145-150$ \\
\hline
\end{tabular}

\section{QUASI-INDUSTRIAL GRADE LASER SOURCES AND BURN-IN EVALUATION}

For industrial applications like silicon photovoltaic cells engraving or high-density micro-electronics, nanosecond or picosecond pulsed regimes are preferred and laser sources based on Master Oscillator Power Amplifier (MOPA) architectures are more relevant. Compared to standard laser oscillators, such laser architectures offer more flexibility to manage pulse duration and repetition rate. A careful management of the signal launching conditions also participates to the reduction of TMI drawbacks as the fundamental mode is preferably excited.

\section{A. Quasi-industrial grade laser sources}

Figure 4 shows a schematic representation of a MOPA architecture. In our experiment, the available pump power was $400 \mathrm{~W}$ and the ns-class seeder emitting at $1030 \mathrm{~nm}$ was able to deliver $12 \mathrm{~ns}$ pulses. The repetition rate was fixed at $250 \mathrm{kHz}$ and average power was tuned up to $4 \mathrm{~W}$. A similar experiment was successfully conducted with another seeder emitting $50 \mathrm{ps} / 2 \mathrm{MHz}$ pulses.

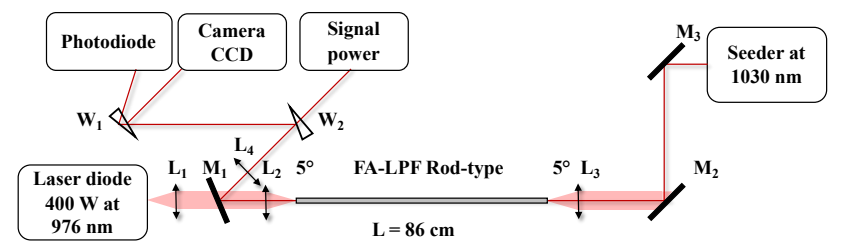

Fig.4. Schematic representation of the experimental MOPA setup. M1, and $\mathrm{M} 2$ are $22.5^{\circ}$ dichroic mirrors (HR1030/HT976); M3 is a HR mirrors at $1030 \mathrm{~nm}$; L1, L2, L3, and L4 are AR-coated lenses and W1 and W2 are wedge plates.

Figure 5 shows the emitted power plotted in regard of the pump power for three different seed powers. The maximum average power was $210 \mathrm{~W}$ limited by available pump power without any observation of TMI on the photodiode. Peak power and energy per pulse were respectively $70 \mathrm{~kW}$ and $0.9 \mathrm{~mJ}$. For all power levels, the mode intensity distributions (see insert in figure 5) as well as the $\mathrm{M}^{2}$ value were measured and compared. No degradation was observed up to maximum power level. 


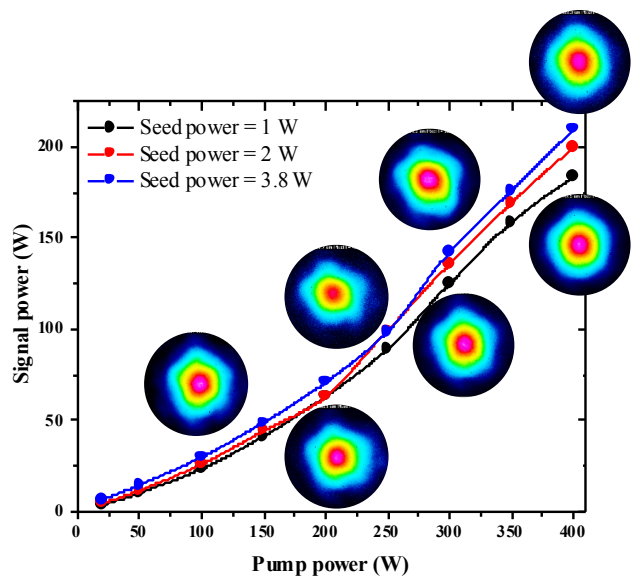

Fig.5. Emitted power versus pump power. Three seed (12ns/250kHz) average powers are compared from 1 to $3.8 \mathrm{~W}$ without TMI observation. Intensity distributions of emitted beams are displayed in inset along the curves.

\section{B. Burn-in evaluation}

For industrial qualification of laser sources, long term running have to be operated. A burn-in test is reported in this section with several outputs. Indeed, for high density microelectronics, pulsed laser sources are associated with Second or Third Generation Harmonic (SHG/THG) stages.

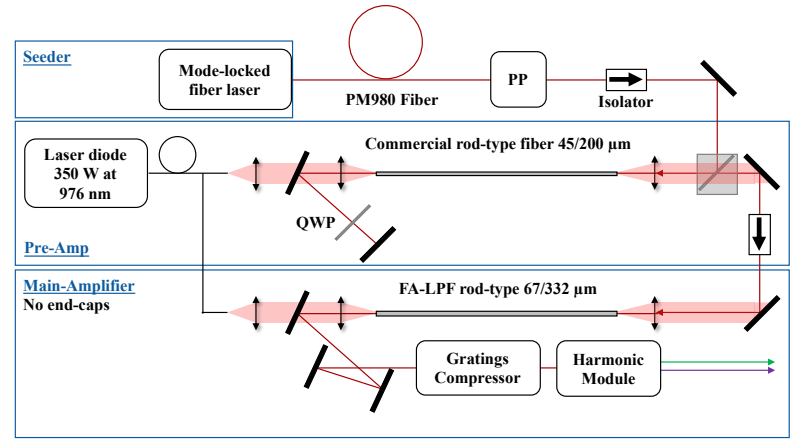

Fig.6. Schematic representation of the laser source used to perform the burn-in tests. A dual amplifier stage femtosecond laser source was associated with a compressor and the SHG module.

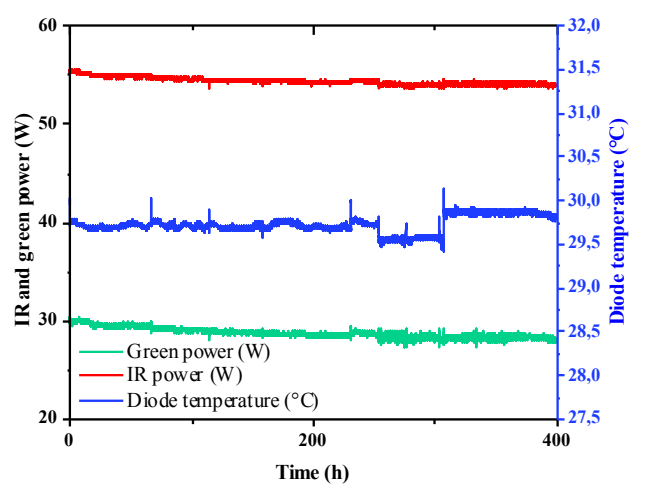

Fig.7. Evolution of the emitted IR and green powers during 400 hours related to the diode temperature fluctuations.
With such devices, monitoring the green output power level is a very severe test as the efficiency of the SHG process strongly depends on the laser peak and average power on one hand and spectral, temporal modal stability on the other hand. Figure 6 shows the device that was used to perform the burnin test over several hundred hours. Figure 7 shows the temporal evolution of both IR and green laser power as well as the diode temperature over 400 hours. The FA-LPF used in this experiment was $75 \mathrm{~cm}$ long and exhibited a core diameter and a mode field diameter of respectively 90 and $67 \mu \mathrm{m}$. The SHG conversion efficiency was $50 \%$ and the measured $\mathrm{M}^{2}$ values were below 1.1 on both axes. During the whole test, the pulse duration was constant and equal to $660 \mathrm{fs}$. The slight drop observed on the average power in the first 300 hours could be due to the photodarkening. The RMS power instability on the last 100 hours (at $2 \sigma$ ) is below $1 \%$ in both IR and green.

\section{PRE-COMPENSATION OF THERMAL LOADING}

Finally, although optical fibers are known to be unsensitive to thermal effects due to their geometrical properties, the LMA faces thermal drawback that gives rise to TMIs. Indeed, as extreme values of core areas are requested to propagate high peak power/energy pulses, refractive index profiles of LMA fibers have to demonstrate very low numerical apertures, i.e. very low refractive index contrast between core and cladding. Regardless of the design, a rough calculation of normalized frequency in a step index-like fiber shows that for a $100 \mu \mathrm{m}$ core diameter, the $\mathrm{V}$ parameter remains below 2.407 while the index contrast between core and cladding is smaller than $2.10^{-5}$. On the other hand, under severe laser conditions, the heat load in the core of a rod type fiber can induce 5 to $10.10^{-5}$ parabolic index contrast that strongly modify the guidance properties, the modal population and consequently modal stability. Postponing the appearance of TMI may imply the thermal pre-compensation of the refractive index map of the structure. Figure 8 illustrates this purpose with the simplest strategy which consists in homogeneously decreasing the refractive index of the core. Beyond the schematic representation in figure 8 , a rod type fiber has been fabricated with a refractive index depression in the core of $3 \cdot 10^{-5}$. The fiber has been drawn with a MFD/core size of $92 / 113 \mu \mathrm{m}$ and characterized on the device shown in figure 4 .

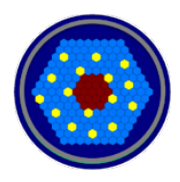

(a)

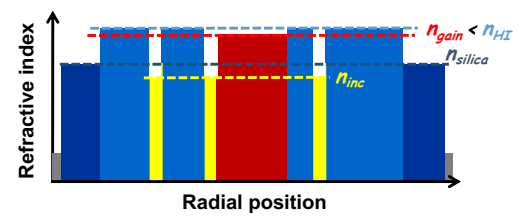

(b)
Fig.8. Schematic representation of (a) the cross-section and (b) refractive index profile of the proposed pre-compensated FA-LPF.

At low pump power level, the fundamental mode leaks into the cladding as the overall index profile act as an antiguide despite the presence of the aperiodic structure in the cladding. Figure 9 shows the evolution of the emitted power versus pump power for several levels of seed power. On the same figure, the intensity distributions of the emitted beams are set in inserts. Until $200 \mathrm{~W}$ of pump power, the laser efficiency is far below the expected one and the intensity distribution of the mode clearly shows a lack of confinement. 
Beyond $200 \mathrm{~W}$ of pump power, we clearly observe an increase of laser efficiency together with an improvement of the intensity distribution of the mode. The latter being slightly pentagonal, this shows that the guidance is now operated by the aperiodic structure. The available pump power being limited at $400 \mathrm{~W}$, it was not possible to measure the TMI threshold during this experiment although the very large core should have made the fiber very sensitive to TMI.

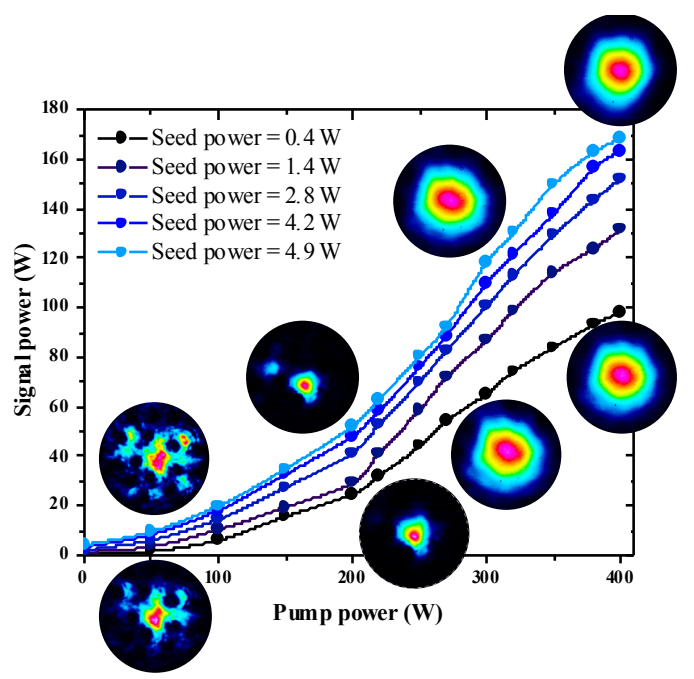

Fig.9. Evolution of the emitted signal power versus incident pump power for five seed power levels (from 0.4 to $4.9 \mathrm{~W}$ ). Inserts depict the far-field intensity distribution of emitted beams for 0.4 and $4.9 \mathrm{~W}$ seed levels at $50 \mathrm{~W}, 200 \mathrm{~W}, 300 \mathrm{~W}, 400 \mathrm{~W}$ pump power levels.

\section{CONCLUSION}

The paper makes a review on Fully-Aperiodic-LargePitch-Fiber. Beyond the concept itself, different generations of fiber samples are described and their performances are discussed, particularly for the link between their structural imperfections and their respective TMI threshold. Some samples among the closest from the model have been successfully characterized in quasi-industrial grade lasers demonstrating exceptional power and modal stability during burn-in and extremely low dependence to photodarkening. Finally, promising testfs have been done with an extremely large core $(\mathrm{MFD}=92 \mu \mathrm{m})$ fibre specially designed to precompensate the thermal loading.

\section{ACKNOWLEDGMENT (Heading 5)}

Authors thank Eolite Systems, Amplitude Systèmes, Thales Optronics SA and Leukos, as well as the Region Nouvelle-Aquitaine for supporting the EATLase project through a multi-annual collaboration agreement and financial support. We also thank the ANRT for supporting the thesis of M-A. Malleville. Authors also thanks Kay Schuster as exleader of the optical fiber team at the IPHT.

\section{REFERENCES}

[1] J. C. Knight, T. A. Birks, P. St. J. Russell, and D. M. Atkin, "All-silica single-mode optical fiber with photonic crystal cladding," Opt. Lett. 21 , pp. 1547-1549 (1996)

[2] W. S. Wong, X. Peng, J. M. McLaughlin, and L. Dong, "Breaking the limit of maximum effective area for robust single-mode propagation in optical fibers," Opt. Lett. 30, pp. 2855-2857 (2005).

[3] J. Limpert, O. Schmidt, J. Rothhardt, F. Röser, T. Schreiber, A. Tünnermann, S. Ermeneux, P. Yvernault, and F. Salin, "Extended single-mode photonic crystal fiber lasers," Opt. Express 14(7), 27152720 (2006).

[4] T. T. Alkeskjold, M. Laurila, L. Scolari, and J. Broeng, "Single-mode ytterbium-doped large-mode-area photonic bandgap rod fiber amplifier," Opt. Express 19, pp. 7398-7409 (2011).

[5] F. Jansen, F. Stutzki, H-J. Otto, M. Baumgartl, C. Jauregui, J. Limpert, and A. Tünnermann, "The influence of index-depressions in corepumped Yb-doped large pitch fibers," Opt. Express 18, pp. 2683426842 (2010).

[6] A. Klenke, S. Hädrich, T. Eidam, J. Rothhardt, M. Kienel, S. Demmler, T. Gottschall, J. Limpert, and A. Tünnermann, "22 GW peak-power fiber chirped-pulse-amplification system," Opt. Lett. 39, pp. 6875-6878 (2014).

[7] M. Laurila, M. M. Jørgensen, J. Lægsgaard, and T. T. Alkeskjold, "Highly Efficient $90 \mu \mathrm{m}$ Core Rod fiber Amplifier Delivering $>300 \mathrm{~W}$ Without Beam Instabilities," in 2013 Conference on Lasers and Electro-Optics - International Quantum Electronics Conference, (Optical Society of America, 2013), paper CJ_3_5.

[8] C. Jauregui, J. Limpert, and A. Tünnermann, "High-power fibre lasers," Nature Photonics 7, pp. 861-917 (2013).

[9] M. N. Zervas, Proc. SPIE 10083, 100830M (2017).

[10] T. Eidam, C. Wirth, C. Jauregui, F. Stutzki, F. Jansen, H.-J. Otto, O. Schmidt, T. Schreiber, J. Limpert, et A. Tünnermann, « Experimental observations of the threshold-like onset of mode instabilities in high power fiber amplifiers », Opt. Express 19, pp.13218-13224 (2011).

[11] H.-J. Otto, F. Stutzki, F. Jansen, T. Eidam, C. Jauregui, J. Limpert, et A. Tünnermann, «Temporal dynamics of mode instabilities in highpower fiber lasers and amplifiers », Opt. Express 20, 15710-15722 (2012).

[12] M. M. Johansen, K. R. Hansen, M. Laurila, T. T. Alkeskjold, et J. Lægsgaard, «Estimating modal instability threshold for photonic crystal rod fiber amplifiers », Opt. Express 21, pp. 15409-15417 (2013).

[13] M-A. Malleville, R. Dauliat, A. Benoît, B. Leconte, D. Darwich, R. du Jeu, R. Jamier, K. Schuster, and P. Roy, "Experimental study of the mode instability onset threshold in high-power FA-LPF lasers," Opt. Lett. 42, 5230-5233 (2017).

[14] R. Dauliat, D. Gaponov, A. Benoit, F. Salin, K. Schuster, R. Jamier, and P. Roy, "Inner cladding microstructuration based on symmetry reduction for improvement of singlemode robustness in VLMA fiber," Opt. Express 21, 18927-18936 (2013).

[15] H-J. Otto, F. Stutzki, N. Modsching, C. Jauregui, J. Limpert, and A. Tünnermann, "2 $\mathrm{kW}$ average power from a pulsed $\mathrm{Yb}$-doped rod-type fiber amplifier," Opt. Lett. 39, pp. 6446-6449 (2014).

[16] H-J. Otto, N. Modsching, C. Jauregui, J. Limpert, and A. Tünnermann, "Impact of photodarkening on the mode instability threshold," Opt. Express 23, pp. 15265-15277 (2015).

[17] R. Dauliat, A. Benoît, D. Darwich, R. Jamier, J. Kobelke, S. Grimm, K. Schuster, and P. Roy, "Demonstration of a homogeneous Yb-doped core fully aperiodic large-pitch fiber laser," Appl. Opt. 55, pp. 62296235 (2016).

[18] C. Jauregui, H-J. Otto, F. Stutzki, J. Limpert, and A. Tünnermann, "Simplified modelling the mode instability threshold of high power fiber amplifiers in the presence of photodarkening," Opt. Express 23, pp. 20203-20218 (2015).

[19] K. Schuster, S. Unger, C. Aichele, F. Lindner, S. Grimm, D.Litzkendorf, J. Kobelke, J. Bierlich, K. Wondraczek, and H. Bartelt, Adv. Opt. Technol. 3, 447 (2014).

[20] A. D. Yablon, J. Lightwave Technol. 28, 360 (2010) 